\title{
A COMUNICAÇÃO EM SAÚDE APROXIMANDO TRABALHADORES DA SAÚDE E ESTUDANTES DE MEDICINA: UMA ESTRATÉGIA PARA O CONTROLE DA DENGUE NO BRASIL
}

\author{
Edlaine Faria de Moura Villela, Wanderson Sant'ana de Almeida, Wallace Antunes Damásio \\ do Nascimento, Luana Kronit Bastos, Gabriel Gonçalves Dutra e Fábio Morato de Oliveira
}

Escola de Medicina, Unidade de Ciências da Saúde da Universidade Federal de Jataí, Goiás, Brasil. edlaine@ufg.br, wandersonalmeida98@gmail.com, damasio.ufg@gmail.com, luanakronit@gmail.com,gdutra.gabriel@gmail.com,

fabiomorato@ufg.br

\begin{abstract}
Resumo. Introdução: É pouco conhecido o processo de orientação e formação dos agentes comunitários de saúde (ACS) para tratar de epidemias. Neste contexto, salienta-se a importância da aproximação entre estudantes de Medicina e ACS para o fortalecimento da educação permanente dos ACS. Objetivo: Promover a educação permanente dos ACS sobre dengue por meio da participação efetiva dos estudantes de medicina para desenvolver habilidades de comunicação dos envolvidos. Método: O método adotado foi o grupo focal com seis ACS de uma Unidade Básica de Saúde (UBS) em Jataí, Goiás, Brasil. O grupo focal foi conduzido por estudantes de medicina, seguido da Análise de Conteúdo. Resultados: Os estudantes observaram que os ACS acreditam que as estratégias comunicacionais adotadas são efetivas e acessíveis. Entretanto, constataram que há falhas comunicacionais a serem sanadas, pois a comunidade continua relutante em por em prática seus conhecimentos, encontrando assim um desafio a ser trabalhado com os ACS ao longo de sua formação acadêmica. Os alunos já começaram a elaborar suas estratégias de ação, com base em conhecimentos construídos sobre comunicação e saúde coletiva. Conclusões: Acredita-se que este trabalho é de grande importância para desenvolver habilidades comunicacionais nos estudantes e para fortalecer seu compromisso com a responsividade social.
\end{abstract}

Palavras-chave: Educação Médica; Habilidades de Comunicação; Agentes Comunitários de Saúde; Grupo Focal.

HEALTH COMMUNICATION APPROACHING HEALTH WORKERS AND MEDICAL STUDENTS: A STRATEGY FOR DENGUE CONTROL IN BRAZIL

Abstract. Introduction: The way how Community Health Workers (CHW) receive training to manage epidemic is almost unknown. In this context, this project addresses the importance of bringing together medical students and $\mathrm{CHW}$ in order to strengthening continuing education of $\mathrm{CHW}$. Objective: To promote the continuing education of $\mathrm{CHW}$ on dengue through the effective participation of medical students to develop communication skills of those involved. Method: The method adopted was the conduction of a focus group with six $\mathrm{CHW}$ in a primary health care centre in a mid-size town of Brazilian Midwest. The focus group was conducted by tutor and students previously trained on dengue prevention and management. Results: Students identified communicational gaps based on what was told by $\mathrm{CHW}$ as correct actions related to dengue control, allowing them to start developing action strategies. This approach not only contributed for continuing education of $\mathrm{CHW}$ but also became a effective transdisciplinary and interprofessional education opportunity for the students involved in the process. CHWs believed their communication strategies about dengue are effective and affordable, however the students detected communication failures and created opportunities to address these misconceptions. Students have already started developing strategies based on knowledge acquired on public health and experienced practices in medical school. Conclusion: We believe this project also offers a great importance for the development of students 'communication skills and increase commitment and social accountability.

Keywords: Medical Education; Communication Skills; Community Health Workers; Focal Group. 


\section{INTRODUÇÃO}

A dengue se tornou um grande problema de saúde pública no Brasil, assim como em outros países. Sua transmissão é essencialmente urbana, onde encontra todos os fatores favoráveis para a sua ocorrência. No Brasil, as condições socioambientais foram favoráveis para a expansão do Aedes aegypti, o qual teve sua reintrodução em 1976 onde não conseguiram de maneira efetiva controlar a propagação (Costa \& Natal, 1998).

A doença foi observada pela primeira vez no estado de Goiás em 1987, no sul do estado. O comportamento sinantrópico e antropofílico desse vetor possibilitou sua expansão geográfica e, em 1990, foi descrito pela primeira vez na capital do estado. Após cinco anos, 59 municípios registravam epidemias de dengue. A partir daí, a doença mostrou uma tendência ascendente, mesmo com as atividades de controle. A transmissão apresenta comportamento cíclico, intercalando anos com altas e baixas incidências (Souza, Silva, \& Silva, 2010).

O Estado de Goiás é constituído de 246 municípios, nos quais as atividades de controle são realizadas de forma descentralizada, em ações do Sistema Único de Saúde (SUS). A expansão da dengue aponta para a necessidade da reestruturação da vigilância epidemiológica, mudança das políticas de controle, inclusão das realidades municipais, gestão ambiental e integração de outros setores da sociedade.

Outra epidemia importante de dengue em Goiás aconteceu em 2005, com 16.725 casos, em 101 municípios. Isso significou um aumento de casos de $307,6 \%$ em relação ao ano anterior, e de $11 \%$ do número de municípios. A transmissão foi contínua em Goiânia, Aparecida de Goiânia, Trindade, Senador Canedo, Rio Verde e Uruaçu (Souza, Silva, \& Silva, 2010).

A prevenção e o controle das doenças transmissíveis indicam a necessidade de uma abordagem interdisciplinar e de inovação estratégica de políticas públicas de saúde. A educação em saúde passa a ser vista não apenas uma mudança de hábitos, ideais e incorporações de conceitos, mas sim como um instrumento para desencadear e fortalecer posturas mais autônomas e politicamente mais efetivas, principalmente nas classes mais desfavorecidas No trabalho diário das equipes de saúde, são os agentes comunitários de saúde (ACS) que possuem um papel de tradutores do universo cientifico para o popular, sendo assim importantes facilitadores do acesso da população aos cuidados de saúde, 
aumentando o alcance da educação em saúde como instrumento modificador de posturas e hábitos (Mialhe, 2011).

O ACS hoje é uma força de trabalho com mais de 200 mil profissionais em todo o país, mas ainda são pouco conhecidas as suas atividades como membro de uma equipe de saúde, sendo que este medeia a relação da equipe com a comunidade. A educação em saúde é uma de suas atividades, mas que nem sempre se mostra clara ou evidente a concepção de educação empregada por esses agentes (Mialhe, 2011). Nota-se então a importância dos ACS em atuação conjunta com a equipe de controle de endemias, não somente efetuando o encaminhamento de casos suspeitos à unidade de atenção primária, mas também nas atuações junto aos domicílios, informando e orientando os moradores sobre a doença, sintomas e riscos, o agente transmissor e medidas de prevenção, chamando a atenção para os criadouros mais comuns na sua área de atuação, na tentativa de estimular os moradores a adotarem ações de prevenção de forma espontânea e rotineira, garantindo o acesso dos agentes de controle de endemias (Brasil, 2009).

No caso específico da Saúde Pública, compreender como as informações chegam aos indivíduos e às comunidades, como elas circulam, como são interpretadas e apropriadas, torna-se um aspecto fundamental na construção de estratégias de prevenção e controle de doenças, como a dengue.

A expansão da dengue aponta para a necessidade da reestruturação da vigilância epidemiológica, mudança das políticas de controle, inclusão das realidades municipais, gestão ambiental e integração de outros setores da sociedade, como o setor comunicacional. Os programas essencialmente centrados em combate químico, com quase nenhuma participação da comunidade, mostraram-se incapazes de conter o vetor, que possui uma alta capacidade de adaptação a novos ambientes. A prevenção e o controle das doenças transmissíveis, como a dengue, indicam a necessidade de uma abordagem interdisciplinar e de inovação estratégica de políticas públicas de saúde.

Ademais, estudos apontam para a contribuição significativa do desenvolvimento de habilidades de comunicação durante a graduação e residência na área da saúde, em contrapartida de uma educação em saúde sem enfoque na comunicação, prejudicando até mesmo para a evolução positiva do quadro clínico do paciente (Curtis et al., 2013). 
Salienta-se assim a importância da aproximação entre os discentes do curso de graduação em Medicina e os agentes comunitários de saúde para que haja o fortalecimento da formação acadêmica. Diante do exposto, justifica-se a realização deste trabalho, pois viabilizará o acompanhamento das intervenções dos ACS na comunidade pelos discentes e futuras estratégias de ação a serem desenvolvidas por esses alunos no município de Jataí/GO, contribuindo assim para a formação dos alunos, incluindo o desenvolvimento de habilidades de comunicação.

\section{METODOLOGIA}

\subsection{População e período de estudo}

A população de estudo foram: os ACS em exercício de sua função no ano de 2018/2019 em uma Unidade Básica de Saúde no município de Jataí/GO; os estudantes de Medicina da Universidade Federal de Jataí, GO, Brasil foram os participantes na execução do grupo focal e análise dos dados, juntamente com a equipe de professores.

\subsection{Grupo focal}

O método adotado para a realização desse estudo foi o grupo focal. A escolha desse método de pesquisa qualitativa pode ser explicada pela intenção de ampliar o olhar do pesquisador e propiciar maior riqueza informacional, conduzindo ao encontro de diferentes percepções acerca de uma prática em educação em saúde que carrega consigo inquietações. Além disso, é um método simples e rápido que consegue ultrapassar a perspectiva individual e atingir uma perspectiva do grupo social (Carlini-Cotrim, 1996). O trabalho foi realizado com agentes comunitários de saúde, os quais ganharam grande importância como agentes de estratégias de linha direta, atuando na vigilância e promoção da saúde.

O grupo focal é bastante popular em pesquisas atualmente, devido à flexibilidade que possui. Esse método apresenta uma literatura substancial sobre como conduzir o estudo em várias disciplinas, como, por exemplo: educação, comunicação, estudos de mídia e psicologia social. No entanto, a literatura é escassa quando a questão é como se analisar os dados resultantes (Silverman, 2004). Essa técnica consiste de pequenos grupos que participam de uma entrevista com um tópico específico entre trinta minutos e duas horas, na qual o entrevistador deverá estimular os participantes da entrevista para obter suas 
respostas (Flick, 2009). Nessa pesquisa, o grupo foi composto por seis ACS. O início da discussão no grupo focal deu-se por meio de um desdobramento do problema dengue, seguido dos tópicos escolhidos.

Os participantes do estudo deram suas dimensões de conhecimento, intervenções e orientações, e os dados foram registrados e gravados para os fins da pesquisa. Em seguida, foi feita a transcrição literal do material verbal coletado durante a discussão em grupo para análise e interpretação dos dados obtidos.

Preparação do roteiro do grupo focal.

No momento de elaboração do roteiro para execução do grupo focal, de acordo com Gil (2009), alguns cuidados devem ser tomados:

- As instruções para o mediador devem ser elaboradas com clareza (como se inicia a entrevista, quanto tempo terá para realiza-la e onde será realizada);

- As questões devem ser elaboradas de forma simples para facilitar a compreensão tanto mediador quanto dos participantes;

- Questões que podem ser vistas como ameaçadoras devem ser elaboradas de maneira que se evite o constrangimento;

- As questões devem conduzir ao engajamento dos participantes, mantendo o interesse no desenvolvimento do grupo focal.

Uma questão importante a ser considerada é a manutenção do foco, evitando discussões de assuntos estranhos aos objetivos do grupo focal. Caso aconteça esse desvio, é necessário mostrar respeito e interesse, mas concluir o tópico e prosseguir com o tema de interesse, lembrando que o mediador deve estimular a conversação, mas não ser o que fala mais no decorrer do processo (Gil, 2009).

Com base nessa fundamentação teórica, foi elaborado um roteiro para condução do grupo focal proposto nesse trabalho, o qual se encontra composto por cinco questões-chave condutoras do grupo focal, baseado no objetivo proposto. Dessas cinco questões-chave, possíveis desdobramentos foram elencados, considerando tópicos que seriam interessantes aparecerem no decorrer da realização do grupo focal. A seguir, encontra-se o roteiro elaborado: 
1. O que é educação em saúde para vocês?

a. Quais são os procedimentos que vocês consideram essenciais para que se alcance a educação em saúde?

b. A teoria encontra-se distante da prática?

2. Quais os temas mais tratados com a comunidade?

a. Qual o vocabulário adotado para se aproximar do público?

b. O ciclo de transmissão da doença é explicado?

3. Como vocês abordam o tema dengue com a comunidade?

a. A abordagem é diferente para cada bairro?

b. A abordagem é feita considerando a idade e o sexo dos moradores?

4. Como enxergam a dengue nos distritos do município?

a. Há diferença de um distrito para outro?

b. Há relação com o nível sócio-econômico?

5. O que vocês acham das campanhas feitas para prevenção e combate da dengue?

a. Quais são os meios de comunicação mais utilizados?

b. O que é mais abordado nas campanhas?

c. São eficientes? São viáveis?

\subsection{Análise dos dados}

A análise e interpretação dos dados foi feita por meio da análise de conteúdo, desenvolvida por Laurence Bardin. Bardin (2010) explica que a análise de conteúdo é a reunião de técnicas de investigação que permitem a interpretação de comunicações presentes em diversas situações cotidianas. Essa interpretação dá-se diante da realização de um trabalho descritivo, objetivo e sistemático, capacitando a compreensão e exploração do tema pelo pesquisador. 
Neste trabalho, foi adotada a análise de conteúdo temática para interpretação do material coletado, pois essa parte da contagem de temas ou itens de significação em uma unidade de codificação, ou seja, pode-se dividir o material verbal em temas principais que emergiram da análise.

Neste trabalho, foi adotada a análise de conteúdo temática para interpretação do material coletado, pois essa parte da contagem de temas ou itens de significação em uma unidade de codificação, ou seja, pode-se dividir o material verbal em temas principais que emergiram da análise. Consiste em salientar núcleos de sentido de uma comunicação existente que signifique de fato alguma coisa para a análise da pesquisa (Silva \& Assis, 2010).

A análise de conteúdo, para melhor compreensão e execução, é feita em três processos: pré-análise, exploração do material e tratamento dos resultados/interpretação (Bardin, 2010). Segue apenas uma breve explanação de cada processo:

- Pré-análise: realização da leitura flutuante (reconhecimento do conteúdo do material), da constituição do corpus (organização para atender quesitos de validade na pesquisa) e reformulação de hipóteses e objetivos (definição da unidade de registro, delimitação do contexto, recortes, categorização, codificação e teorias que embasarão a análise);

- Exploração do material: análise do texto mediante as categorias formadas na pré-análise;

- Tratamento dos resultados e interpretação: os resultados são tratados para serem válidos e significativos, operações estatísticas podem ser aplicadas. As interpretações geradas podem conduzir a novos olhares teóricos.

\subsection{Aspectos éticos}

O estudo foi enviado, antes de sua execução, à Secretaria Municipal de Saúde de Jataí/GO e ao Comitê de Ética em Pesquisa da UFG Regional Jataí, obtendo aprovação para execução.

Os ACS que concordaram em participar do estudo assinaram um Termo de Consentimento Livre e Esclarecido, nos termos da Resolução 466/12 do Conselho Nacional de Ética em Pesquisa.

\section{DESENVOLVIMENTO}

Diante da realização deste trabalho, obtiveram-se informações que permitiram aos pesquisadores envolvidos estabelecer uma aproximação do universo dos ACS que 
convivem diariamente com a problemática dengue. A partir da descrição dos saberes coletados, foi feita uma análise temática e interpretação para compreender a evolução do quadro da doença no município e como os ACS podem contribuir de maneira mais efetiva.

As informações coletadas pelos estudantes durante todo esse processo foram: falas, opiniões, discursos, atitudes, comportamentos e conhecimentos sobre dengue. Os participantes do estudo expuseram suas dimensões de conhecimento, e os dados foram registrados e gravados para fins de pesquisa. Em seguida, foi feita a transcrição literal do material verbal coletado durante a discussão em grupo para análise e interpretação dos dados obtidos.

Quanto à categorização, foram formuladas três categorias:

Categoria 1: A comunicação frente o desinteresse comunitário

- Curiosidade em saberes sobre a dengue

- A perda do medo epidemiológico

- A eficácia comunicacional das campanhas

- Ausência de ações práticas de prevenção

Categoria 2: Dificuldades encontradas na rotina de combate à dengue

- Falta de comprometimento qualitativo no serviço prestado (perfil quantitativo, periodicidade)

- Falta de profissionais e integração entre eles

- Relação médico-sociedade-ACS

- Sugestão de ações punitivas e a impessoalidade entre agentes e população

Categoria 3: Agravantes e problemas secundários observados

- Falta de esgoto

- Consequências ambientais do uso do fumacê

A categoria 1, "A comunicação frente o desinteresse comunitário", chamou atenção durante a execução do projeto. Os ACS afirmaram que a comunicação sobre dengue é efetiva para a comunidade jataiense, como as campanhas pontuais que são realizadas, por exemplo. No entanto, os ACS mencionaram que não há o desenvolvimento contínuo de ações efetivas de prevenção da dengue, apenas de controle. 
Outro aspecto relevante que foi salientado pelos trabalhadores de saúde é o desinteresse dos cidadãos sobre o tema, e ainda relataram que este desinteresse se deve ao fato das pessoas não mais se sentirem amedrontadas quando o assunto é a doença em questão.

Cabe nesse contexto a corroboração de Araújo (2007), a qual afirma que a presença de muita informação não é garantia de saúde, visto que as pessoas recebem informações variadas sobre dengue, mas nem sempre conseguem se apropriar das mesmas para uso em seu cotidiano, por estar em uma linguagem inacessível ou por simplesmente não ser uma informação útil para a população aprimorar comportamentos e hábitos em relação à prevenção.

A divulgação rápida geralmente baseia-se em informação incompleta ou incorreta. Essa informação pouco fundamentada acaba por reduzir a credibilidade das instituições governamentais. Conforme WHO (2004), para que isso não ocorra, é preciso que a gestão conquiste a confiança do público, comunicando a complexidade do caso, incertezas e riscos à saúde possíveis.

Os estudantes observaram que os ACS acreditam que as estratégias comunicacionais adotadas são efetivas e acessíveis. Entretanto, constataram que há falhas comunicacionais a serem sanadas, pois a comunidade continua relutante em por em prática seus conhecimentos, encontrando assim um desafio a ser trabalhado com os ACS ao longo de sua formação acadêmica, em busca de novas estratégias de ação para o combate da doença. Donalisio (1999) traz que nenhuma municipalização pode apresentar bons resultados no controle de uma epidemia se a instância regional não estiver solidificada e equipada suficientemente, ou seja, é necessário garantir nesse processo que haja recursos suficientes para que as esferas regionais consigam identificar perfis epidemiológicos de maneira rápida e efetiva para comunicar as equipes locais.

Os alunos já elaboraram suas estratégias de ação, com base em conhecimentos construídos sobre comunicação e saúde coletiva na universidade e nas práticas vivenciadas nas UBS. A primeira estratégia, já executada, foi a organização de um encontro pelos alunos do curso de Medicina, destinado para os ACS e para a comunidade atendida pela UBS em questão, além de profissionais de saúde interessados em participar.

O evento foi composto das seguintes atividades: palestra, roda de conversa, teatro, barraca tira-dúvidas. Ademais, foi construído um mural informativo sobre a doença para fixação na 
UBS (via de transmissão, sintomas, tratamento) e situações-problema sobre o tema foram elaboradas para os ACS proporem estratégias de ação. Todas as atividades foram previamente elaboradas e executadas pelos alunos, sob supervisão do professor responsável.

Percebe-se assim que o objetivo proposto foi alcançado, pois a parceria entre estudantes de Medicina e ACS contribuiu principalmente: para a formação adequada dos estudantes, no que tange ao desenvolvimento das habilidades de comunicação; e para a educação permanente dos ACS por meio do trabalho conjunto dos estudantes de medicina envolvidos sobre o tema dengue e outras problemáticas relevantes que vieram à tona.

\section{CONCLUSÕES}

Os alunos de medicina adquiriram competências e habilidades comunicacionais ao elaborarem estratégias de intervenção de acordo com as lacunas comunicacionais identificadas no grupo focal com os ACS, com o propósito de apresentar para estes trabalhadores da saúde novas possibilidades de abordagem sobre o tema Dengue, considerando possíveis desdobramentos para outros temas que envolvem problemas de saúde coletiva, enfrentados pelo Sistema Único de Saúde (SUS).

Com o encontro realizado pelos professores e estudantes para os ACS, os estudantes puderam ser capazes de aprender metodologias ativas para trabalhar temas relevantes na área de saúde com os trabalhadores de saúde, de forma efetiva e horizontal. O próximo passo foi avaliarmos como o acompanhamento do trabalho dos ACS pôde contribuir para a formação dos estudantes de Medicina. Por fim, trazemos uma citação de Yach (1992, p. 611), feita por Carlini-Cotrim (1996), que fundamenta a escolha da metodologia e da técnica de interpretação apresentadas neste trabalho:

"O profissional da 'nova' saúde pública seria aquele treinado em países em desenvolvimento numa perspectiva integrada, onde assuntos como antropologia, sociologia e epidemiologia, entre outros, seriam intrinsecamente articulados num mesmo armamentário."

Essa pessoa estaria então apta a usar a melhor mistura de métodos e disciplinas quando requisitada a abordar problemas. Para se alcançar isso, no entanto, será necessário esforço considerável, desde que seja percebido por vários 'territorialistas' das disciplinas como uma ameaça. Mas os ganhos à saúde pública devem ser enfatizados”. Assim, será possível transpor os resultados positivos em metodologias de novos projetos com enfoque em temas 
relevantes de saúde coletiva, trazendo à tona a valorização do desenvolvimento das habilidades de comunicação durante a graduação e a importância de se fortalecer o compromisso com a educação permanente em saúde para se alcançar, de fato, a socialização de conhecimento construído e a famigerada responsividade social.

\section{REFERÊNCIAS}

Araújo, I. S., Cardoso, J. M. (2007). Comunicação e saúde. Rio de Janeiro: FIOCRUZ.

Bardin, L. (2010). Análise de conteúdo. Tradução de Luís Antero Reto e Augusto Pinheiro. Lisboa: Edições 70.

Brasil. Ministério da Saúde. Secretaria de Vigilância em saúde. (2009). Diretrizes Nacionais para a Prevenção e Controle de Epidemias de Dengue. Brasília: Ministério da Saúde. Disponível em:<http://www.saude.caop.mp.pr.gov.br/arquivos/File/dengue/livroDiretrizes.pdf>. Acesso em: 04.jun.2011.

Carlini-Cotrim, B. (1996). Potencialidades da técnica qualitativa grupo focal em investigações sobre abuso de substâncias. Revista de Saúde Pública, 30(3), 285-293.

Costa, A. I. P., \& Natal, D. (1998). Distribuição espacial da dengue e determinantes socioeconômicos em localidade urbana no Sudeste do Brasil. Revista de Saúde Pública, 32(3), 232-6. Disponível em: <http://www.scielo.br/pdf/rsp/ v32n3/p232-236.pdf.>. Acesso em: 04.jun.2011.

Curtis, J. R. et al. (2013). Effect of Communication Skills Training for Residents and Nurse Practitioners on Quality of Communication With Patients With Serious Illness. JAMA., 310(21), 2271-2281.

Donalísio, M. R. (1999). O dengue no espaço habitado. São Paulo: HUCITEC.

Flick, U. (2009). Introdução a pesquisa Qualitativa. 3. ed. Porto Alegre: Artmed.

Gil, A. C. C. (2009). Métodos e técnicas de pesquisa social. 6. ed. São Paulo: Atlas.

Mialhe, F. L. (2011). O agente comunitário de saúde: praticas educativa. Campinas: Editora da Unicamp.

Silva, J. R. de S., \& Assis, S. M. B. (2010). Grupo focal e análise de conteúdo como estratégia metodológica clínica-qualitativa em pesquisas nos distúrbios do desenvolvimento. Cadernos de Pós-Graduação em Distúrbios do Desenvolvimento, 10(1), 146-152.

Silverman, D. (2004). Qualitative research - theory, method and practice. 2. ed. Sage: London.

Souza, S. S., Silva, I. G., \& Silva, H. H. G. (2010). Associação entre incidência de dengue, pluviosidade e densidade larvária de Aedes aegypti, no Estado de Goiás. Revista da Sociedade Brasileira de Medicina Tropical, 43(2), 152-15.

WHO. (2004). Outbreak communication - best practices for communicating with the public during an outbreak. Singapura: WHO. Disponível em: $\quad<\quad$ http://www.who. int/csr/resources/publications/WHO_CDS_2005_32web.pdf>. Acesso em: 21.jul.2015. 